This item was submitted to Loughborough's Research Repository by the author.

Items in Figshare are protected by copyright, with all rights reserved, unless otherwise indicated.

\title{
Time-to-collision analysis of pedestrian and pedal-cycle accidents for the development of autonomous emergency braking systems
}

PLEASE CITE THE PUBLISHED VERSION

https://doi.org/10.1016/j.aap.2018.02.028

\section{PUBLISHER}

(C) Elsevier

VERSION

AM (Accepted Manuscript)

\section{PUBLISHER STATEMENT}

This work is made available according to the conditions of the Creative Commons Attribution-NonCommercialNoDerivatives 4.0 International (CC BY-NC-ND 4.0) licence. Full details of this licence are available at: https://creativecommons.org/licenses/by-nc-nd/4.0/

\section{LICENCE}

CC BY-NC-ND 4.0

\section{REPOSITORY RECORD}

Lenard, James, Ruth Welsh, and Russell Danton. 2018. "Time-to-collision Analysis of Pedestrian and Pedalcycle Accidents for the Development of Autonomous Emergency Braking Systems”. Loughborough University. https://hdl.handle.net/2134/32144. 


\title{
Time-to-collision analysis of pedestrian and pedal-cycle accidents for the development of autonomous emergency braking systems
}

\author{
James Lenard $^{\mathrm{b}, \mathrm{a}, *}$, Ruth Welsh ${ }^{\mathrm{a}}$, Russell Danton ${ }^{\mathrm{c}}$ \\ ${ }^{a}$ Loughborough University, Loughborough, UK \\ ${ }^{b}$ Datarye Ltd, Royal Leamington Spa, UK \\ ${ }^{c}$ GBB, Crown House, Burnley, UK
}

\begin{abstract}
The aim of this study was to describe the position of pedestrians and pedal cyclists relative to the striking vehicle in the three seconds before impact. This information is essential for the development of effective autonomous emergency braking systems and relevant test conditions for consumer ratings. The UK RAIDS-OTS study provided 175 pedestrian and 127 pedal-cycle cases based on in-depth, at-scene investigations of a representative sample of accidents in 2000-2010. Pedal cyclists were scattered laterally more widely than pedestrians ( $90 \%$ of cyclists within around \pm 80 degrees compared to \pm 20 degrees for pedestrians), however their distance from the striking vehicle in the seconds before impact was no greater (90\% of cyclists within 42 metres at three seconds compared to 50 metres for pedestrians). This data is consistent with a greater involvement of slow moving vehicles in cycle accidents. The implication of the results is that AEB systems for cyclists require almost complete 180 degree side-to-side vision but do not need a longer distance range than for pedestrians.
\end{abstract}

Keywords: time-to-collision, pedestrian, pedal cycle, autonomous emergency braking, AEB sensors, pre-impact location

\section{Introduction}

According to the World Health Organisation pedestrians and cyclists account for $30 \%$ of road traffic fatalities in Europe [1]. With the further encouragement for a modal shift towards cycling and walking, the protection of vulnerable road users remains a

\footnotetext{
${ }^{*}$ Corresponding author

Email address: j.a.lenard@datarye.com (James Lenard)

URL: orcid.org/0000-0002-5084-0484 (James Lenard)
} 
key road safety objective. To this end, autonomous emergency braking (AEB) systems are under continuous development. These are designed to take action on behalf of the driver when the likelihood of an interaction with a cyclist or pedestrian is detected. It is important that such systems are able to perform adequately in a range of scenarios where conflict occurs between a passenger car and a cyclist or pedestrian.

A number of previous studies used real-world data to describe common accident scenarios for cyclist collisions with passenger cars, however these did not report on the relative positioning of the vehicle and vulnerable road user in the moments before impact [2, 3, 4]. An analysis of German in-depth accident data found that half of all pedestrians were detectable three seconds before the accident but for those considered obscured, the time fell to below one second [5]. This study raised the question whether time-to-collision (TTC), vehicle speed or both should be varied in AEB test procedures. A Swedish review of 243 pedestrian accidents in the same German database found that all but one of the 57 serious and fatal casualties were within a field of view of 40 degrees ( \pm 20 degrees) and a range of 20 metres [6]. In another study analysing approach patterns, it was concluded that the time-to-collision for car-to-bicycle nearmiss events was significantly longer than that for car-to-pedestrian events [7].

A recent large-scale naturalistic driving study conducted in Europe gathered information of safety-critical events involving passenger cars and pedal cycles [8]. Of 36 incidents flagged by a forward collision warning system, only three were found on review to be genuine near-crashes, each a head-on scenario. Compounding the low sample size, the use of a forward-looking system necessarily precluded the detection of lateral incidents.

The general purpose of the present study was to add to this real-world knowledge base and thereby contribute to the further development of effective AEB systems and relevant test conditions [9]. The circumstances of real accidents are described, in particular the requirement on the sensing system to detect pedestrians and pedal cycles at their distance and angle relative to the striking vehicle in the seconds immediately before impact, including when the driver's line of sight may have been obstructed by intervening vehicles or fixed objects. The specific aim of the paper was to specify the position of pedestrians and pedal cyclists relative to the striking vehicle at one, two and three seconds before impact based on in-depth accident data from Great Britain.

\section{Material and methods}

The results presented in this paper were based on the On-the-Spot module of the Road Accident In-depth Studies project (RAIDS-OTS) commissioned by the UK De- 
Table 1: Accident severity level for pedestrians and pedal cycles.

Pedestrian Pedal cycle

\begin{tabular}{lrrrr} 
& $\mathrm{n}$ & $\%$ & $\mathrm{n}$ & $\%$ \\
\hline Fatal & 10 & 6 & 1 & 1 \\
Serious & 54 & 31 & 30 & 24 \\
Slight & 108 & 62 & 83 & 65 \\
No injury & 3 & 2 & 12 & 9 \\
\hline Total & 175 & 100 & 127 & 100 \\
\hline
\end{tabular}

partment for Transport and the Highways Agency from 2000 to 2010 [10, 11, 12]. The RAIDS-OTS database contains detailed information from at-scene investigations of a random sample of road traffic accidents reported to the police. The information was collected by two research institutes whose teams operated rotating eight-hour shifts seven days per week in two sample regions, South Nottinghamshire and Thames Valley, and attended the scene of accidents along with the police and emergency services. Each team aimed for 250 accidents per year, ultimately completing 4,744 cases. The sample regions were designed to be nationally representative and the On-the-Spot study was the primary source of in-depth information on accident causation used by the Department for Transport to guide national policy-making during this period. Data collection was suspended in 2010 for a period of restructuring and progressively resumed from 2012 to 2016.

The RAIDS-OTS study sampled accidents involving all types of vehicles. The analysis in this paper focussed on collisions between passenger cars and two classes of vulnerable road users: pedestrians and pedal cyclists. The selection criteria for the inclusion of cases were (a) the vulnerable road user was struck by a passenger car, (b) the car was moving forwards at impact, (c) initial contact was to the front or side of the car and (d) the car was not out of control due to a preceding impact. Some cases with inadequate documentation were excluded from the final analysis, e.g. minor incidents where the accident scene was entirely cleared before arrival of the emergency services and research team. This filtering process yielded 175 pedestrians and 127 pedal cyclists from the 4,744 accidents on file; of these, 64 pedestrians (37\%) and 31 cyclists $(25 \%)$ were killed or seriously injured (Table 1).

The observations and measurements taken at the scene of the accident by the specialist research teams were supplemented where possible by interviews with involved parties and relevant extracts of police and medical reports. The case files for the selected accidents were reviewed in detail to ensure a consistent methodology with full exploitation of the source material. The paths of the road users involved (passenger 
Table 2: Age-sex grouping of pedestrians and pedal cyclists as proxy for target size. Pedestrian Pedal cycle

\begin{tabular}{lrrrr} 
& \multicolumn{2}{c}{ Pedestrian } & \multicolumn{2}{c}{ Pedal cycle } \\
& $\mathrm{n}$ & $\%$ & $\mathrm{n}$ & $\%$ \\
\hline 0-7 years & 23 & 13 & 1 & 1 \\
8-15 years & 47 & 27 & 39 & 31 \\
Adult female & 41 & 23 & 13 & 10 \\
Adult male & 64 & 37 & 67 & 53 \\
Unknown & 0 & 0 & 7 & 6 \\
\hline Total & 175 & 100 & 127 & 100 \\
\hline
\end{tabular}

cars, pedal cycles and pedestrians) in the seconds before impact were generally well documented. The vehicle speed recorded on the database prior to impact or emergency action (if applicable) was extrapolated backwards in time for up to three seconds using constant-acceleration kinematics, i.e. steady braking, constant speed or steady acceleration, consistent with eye-witness reports and traffic conditions at the time and place of the accident. The location of the vulnerable road user relative to the striking vehicle was recorded in a supplementary dataset using a co-ordinate system based on the longitudinal and lateral axes of the striking vehicle. The methodology was harmonised with previous studies as far as could be ascertained [13, 6].

The collection, handling and storage of data in the RAIDS project were subject to strict, high-level ethical and security provisions, exceeding those specified by the UK Data Protection Act 1998, the Cabinet Office's Mandatory Minimum Guidelines and Section 251 of the NHS Act 2006. These were established in liason with security consultants and regularly tested by independent auditors during data collection. In essence only anonymised information was taken from police premises, hospitals and coroners; all information recorded at the site of accident and vehicle examinations was also anonymised during processing of the case and any residual material physically destroyed. These ethical and information governance measures, which have been described in further detail $[14,10]$, protected the right to confidentiality and constituted a precondition to undertaking this type of empirical study.

\section{Results}

A grouping by age and sex for the samples of pedestrians and cyclists is shown in Table 2. These age-sex categories were used as a proxy for target size in the leadup to the introduction of the EuroNCAP AEB pedestrian test $[15,16]$. This table suggests that cyclists as a whole present a larger target for vehicle-mounted AEB sensors than pedestrians: a lower proportion of cyclists were in the smallest group, 


\begin{tabular}{lrr} 
& \multicolumn{2}{c}{ Table 3: Ve } \\
Vehicle & $\mathrm{n}$ & $\%$ \\
\hline Straight ahead & 152 & 87 \\
Turning & 23 & 13 \\
Total & 175 & 100 \\
\hline
\end{tabular}

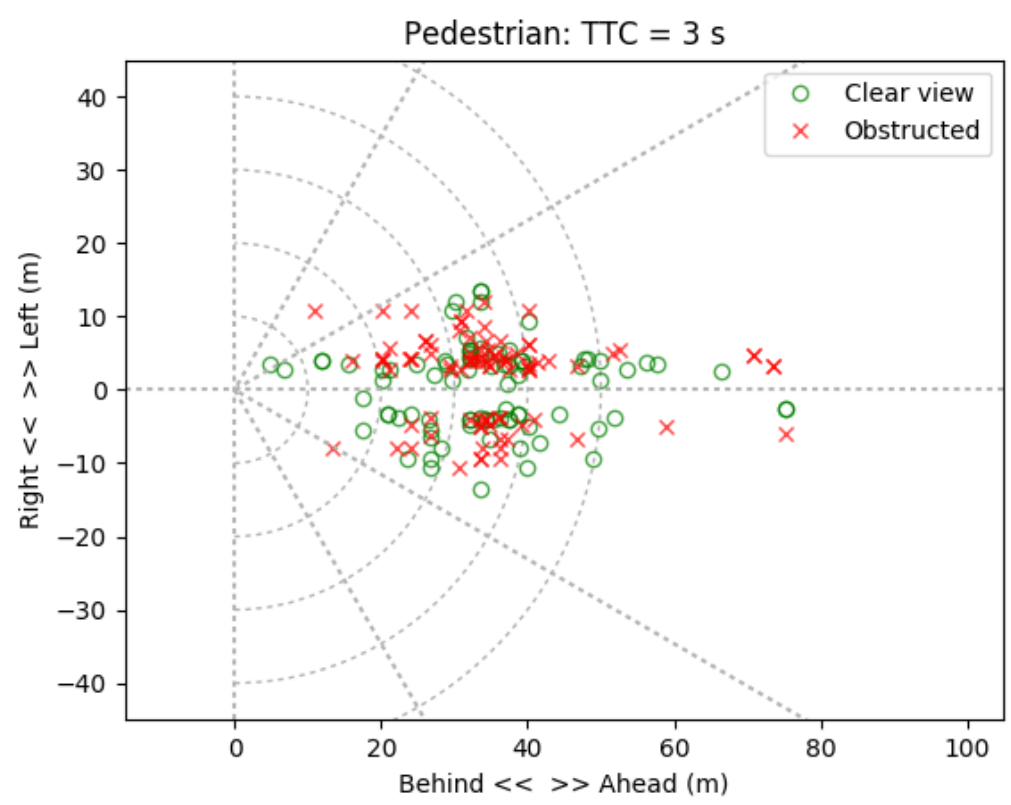

Figure 1: Position of pedestrian relative to car at TTC $=3 \mathrm{~s}$.

young children 0-7 years old (one cyclist compared to $13 \%$ of pedestrians), and a higher proportion were in the largest group, adult males (53\% of cyclists compared to $37 \%$ of pedestrians).

Most vehicles in pedestrian accidents (87\%) were travelling straight ahead rather than turning at an intersection (Table 3). This was determined prior to the introduction by EuroNCAP of straight-line test conditions for pedestrian AEB.

The location of the 175 pedestrians relative to the striking vehicle in the three seconds before impact is shown in Figures 1-3. Cases in which the driver had a clear view of the pedestrian at that moment of time are distinguished from those in which the driver's line of sight was thought to be obstructed, typically by a vehicle or roadside object. The scale of the $\mathrm{X}$ and $\mathrm{Y}$ axes varies in these three figures to accommodate the diminishing range of values for lower times to collision. The number of cases in each 


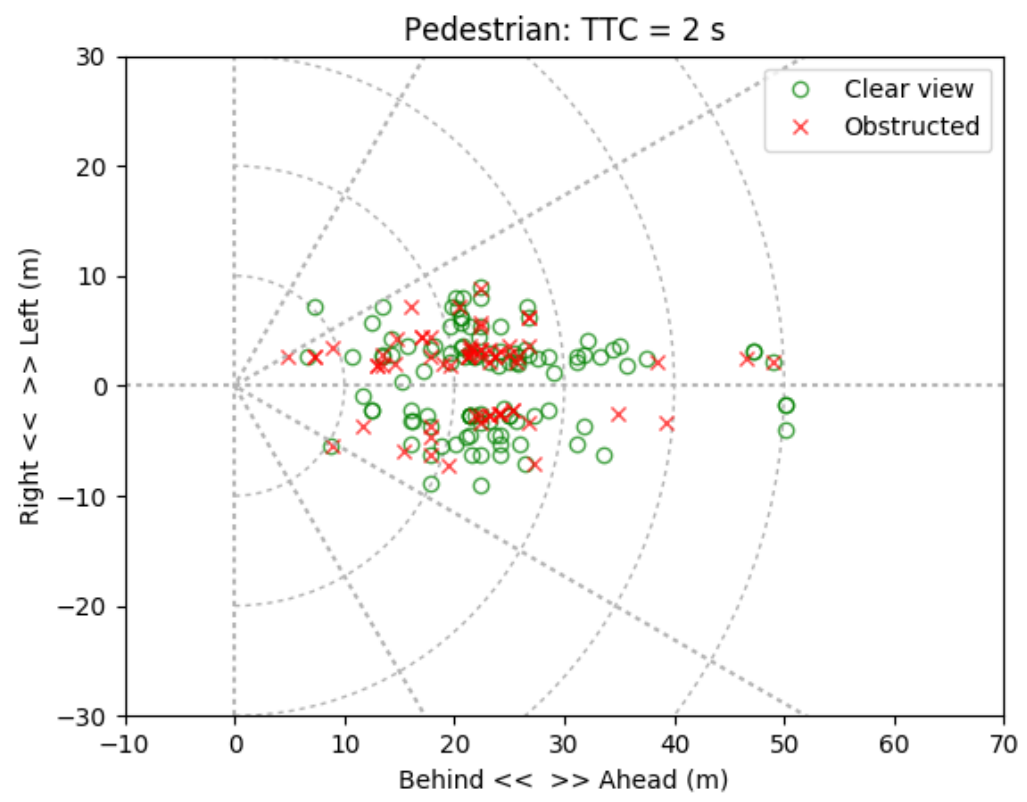

Figure 2: Position of pedestrian relative to car at TTC $=2 \mathrm{~s}$.

cell (10 m by $30 \mathrm{deg})$ marked out by the radial guidelines are detailed in Tables 5-7 (Appendix).

In order to provide an overall impression of the paths of individual pedestrians relative to the striking vehicle in the three seconds before impact, Figure 4 shows the points in Figures 1-3 joined by lines. It can be seen that most paths lie entirely within thirty degrees (each side) of the longitudinal axis of the striking vehicle. The density of lines is inevitably very high when so many cases are presented in a single figure, particularly close to the origin (point of impact); for this reason an animated version of Figure 4 is also provided in which each path is individually highlighted (Supplementary Materials).

Turning to pedal-cycle accidents, Table 4 details the path of the bicycle relative to the path of the car before impact or emergency braking. In 25 cases (20\%) they were travelling in the same direction; in most of these (18) the vehicle was travelling straight ahead, i.e. overtaking. There were 19 cases (15\%) in which the car and bicycle approached from opposite directions; in most of these (15) the car turned across the path of the cyclist. There were 76 cases $(60 \%)$ in which the car and bicycle were on cross-intersection paths before impact; in a high number of these (67) the cyclist was 


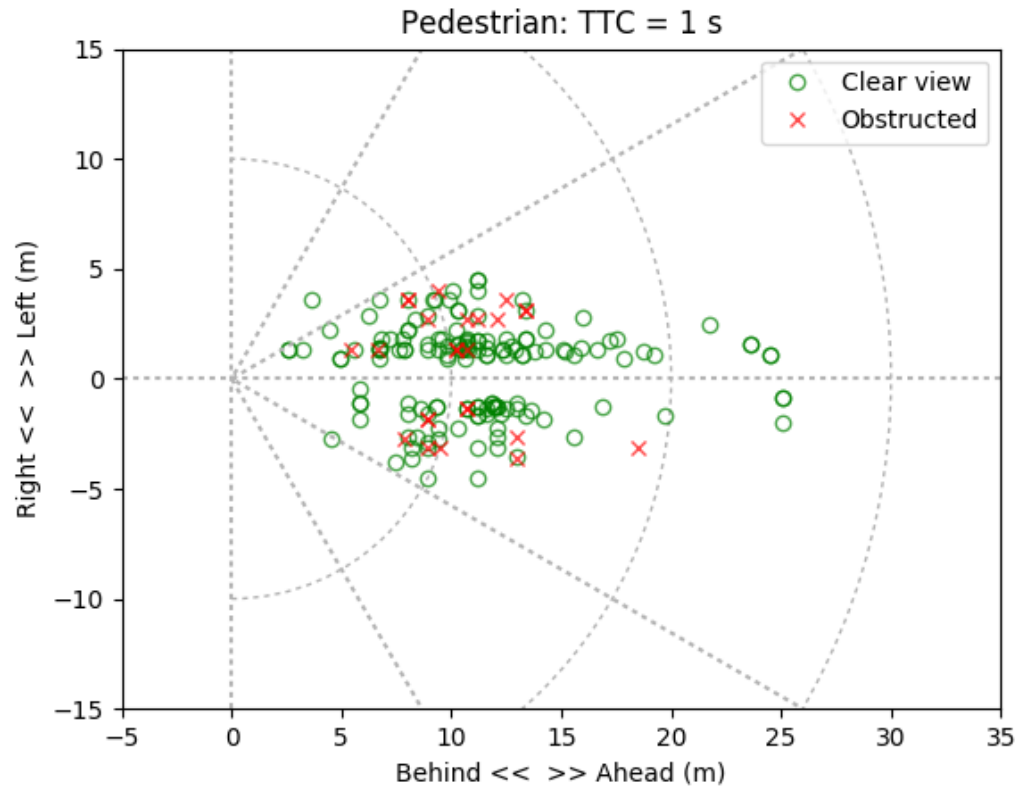

Figure 3: Position of pedestrian relative to car at TTC $=1 \mathrm{~s}$.

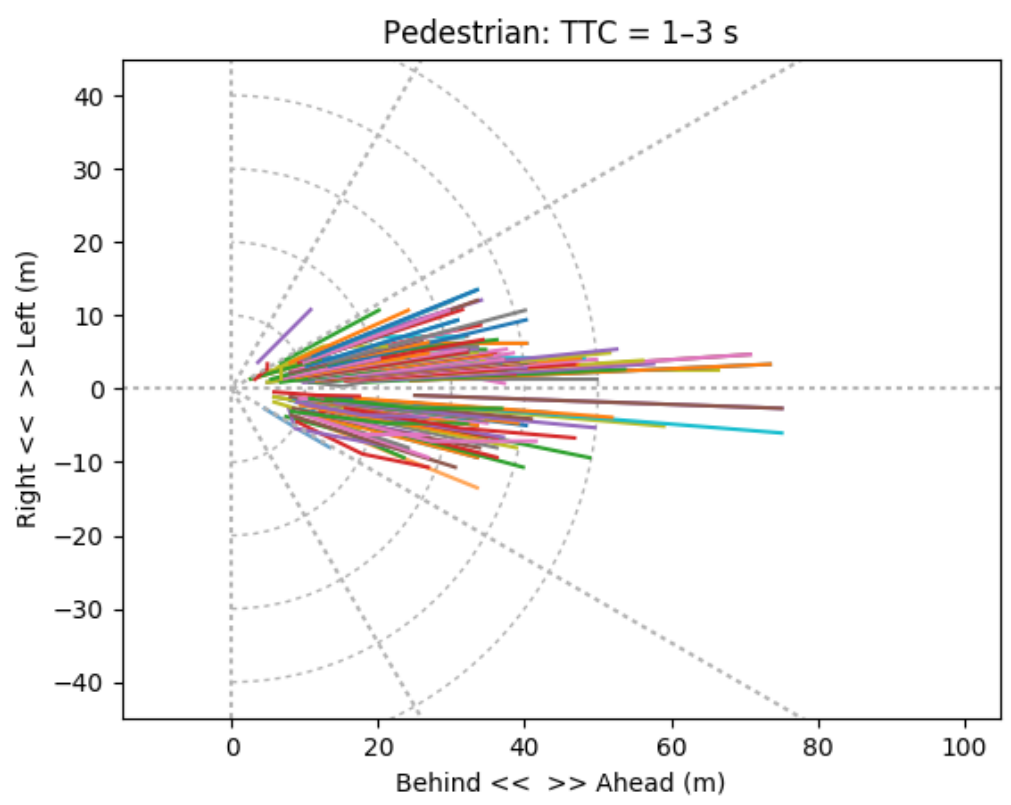

Figure 4: Position of pedestrian relative to car at TTC $=3,2,1 \mathrm{~s}$. 
Table 4: Orientation of bicycle travel direction relative to car before impact.

\begin{tabular}{lcrr} 
Bicycle $\uparrow$ Car $\Uparrow$ & Paths & $\mathrm{n}$ & $\%$ \\
\hline Same direction & $\uparrow \Uparrow$ & 25 & 20 \\
Opposite direction & $\Uparrow \downarrow$ & 19 & 15 \\
Bicycle from left & $\rightarrow \Uparrow$ & 34 & 27 \\
Bicycle from right & $\Uparrow \leftarrow$ & 42 & 33 \\
Other & & 7 & 6 \\
\hline Total & & 127 & 100 \\
\hline
\end{tabular}

on a straight-line course and the car driver intended to proceed ahead (39) or turn (28).

The location of the 127 pedal cyclists relative to the striking vehicle in the three seconds before impact is shown in Figures 5-7. As for the pedestrians above, cases are distinguished according to whether the driver's line of sight to the cyclist was clear or obstructed and the scale of the $\mathrm{X}$ and $\mathrm{Y}$ axes is decreased as the time to collision decreases. Likewise, the number of cases in each cell ( $10 \mathrm{~m}$ by $30 \mathrm{deg}$ ) marked out by the radial guidelines are enumerated in Tables 8-10 (Appendix).

It is apparent that the pedal cycles were scattered across a wider range of angles than the pedestrians, i.e. not so highly concentrated alongside the longitudinal axis of the striking vehicle, as shown in Figure 8 where the path of each cyclist is formed by lines between the points in Figures 5-7. An animated version of Figure 8 highlights the bicycle paths individually (Supplementary Materials).

The extent to which the pedestrians and pedal cyclists were located at angles away from the longitudinal axis of the striking vehicle is quantified in Figure 9 in the form of empirical (non-idealised) cumulative distribution curves derived directly from the data presented in Figures 1-3 and Figures 5-7. No distinction is made between angles left or right of the longitudinal axis, i.e. the plot is based on the absolute value of the angle of the vulnerable road user. The figure shows that while $90 \%$ of pedestrians were well within \pm 25 degrees of the longitudinal axis, this range only encompassed about $50 \%$ of pedal cyclists; $75 \%$ of cyclists were within around 50-60 degrees while 90\% were within around 75-85 degrees, an almost complete side-to-side lateral distribution.

Figure 10 presents empirical cumulative distribution curves for pedestrians and pedal cyclists aggregated across time-to-collision (TTC $=1-3 \mathrm{~s}$ ) but disaggregated according to whether the driver's line of sight to the vulnerable road user was clear or obstructed. For pedestrians, the curves are almost identical, indicating that the distribution of obscured pedestrians as a function of angle was the same as for visible pedestrians. For cyclists, 50\% of visible cases were within 20 degrees of the vehicle's longitudinal axis compared to 30 degrees for obscured cases, $75 \%$ of both visible and 


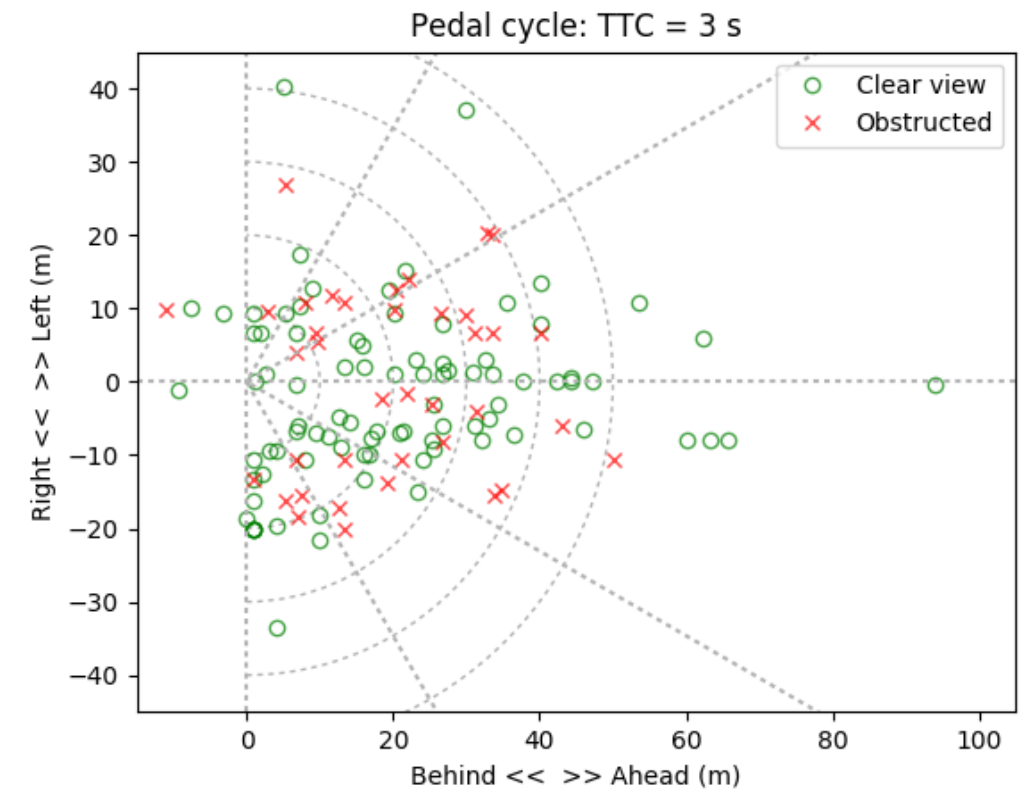

Figure 5: Position of bicycle relative to car at TTC $=3 \mathrm{~s}$.

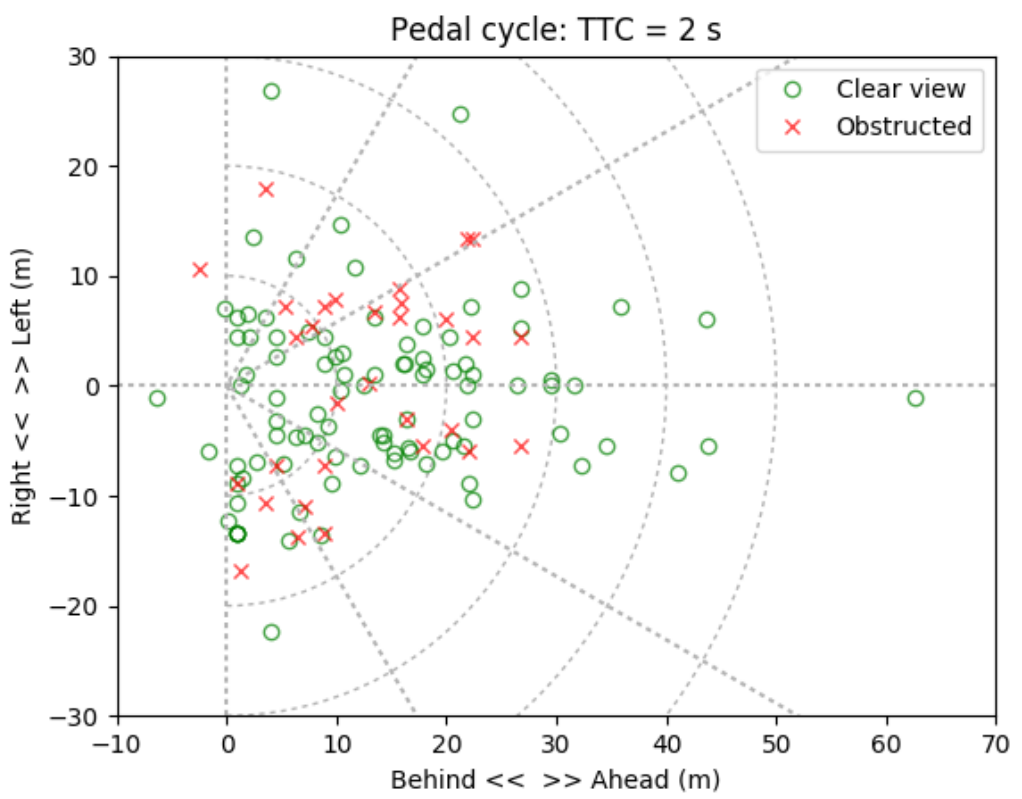

Figure 6: Position of bicycle relative to car at TTC $=2 \mathrm{~s}$. 


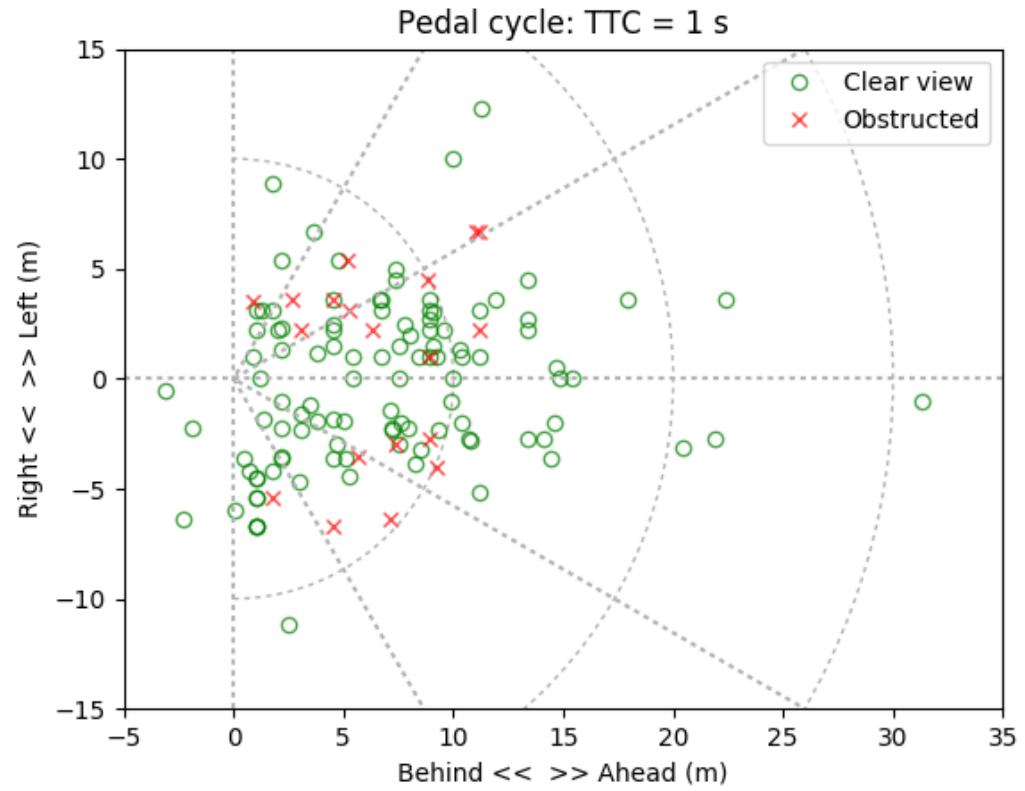

Figure 7: Position of bicycle relative to car at TTC $=1 \mathrm{~s}$.

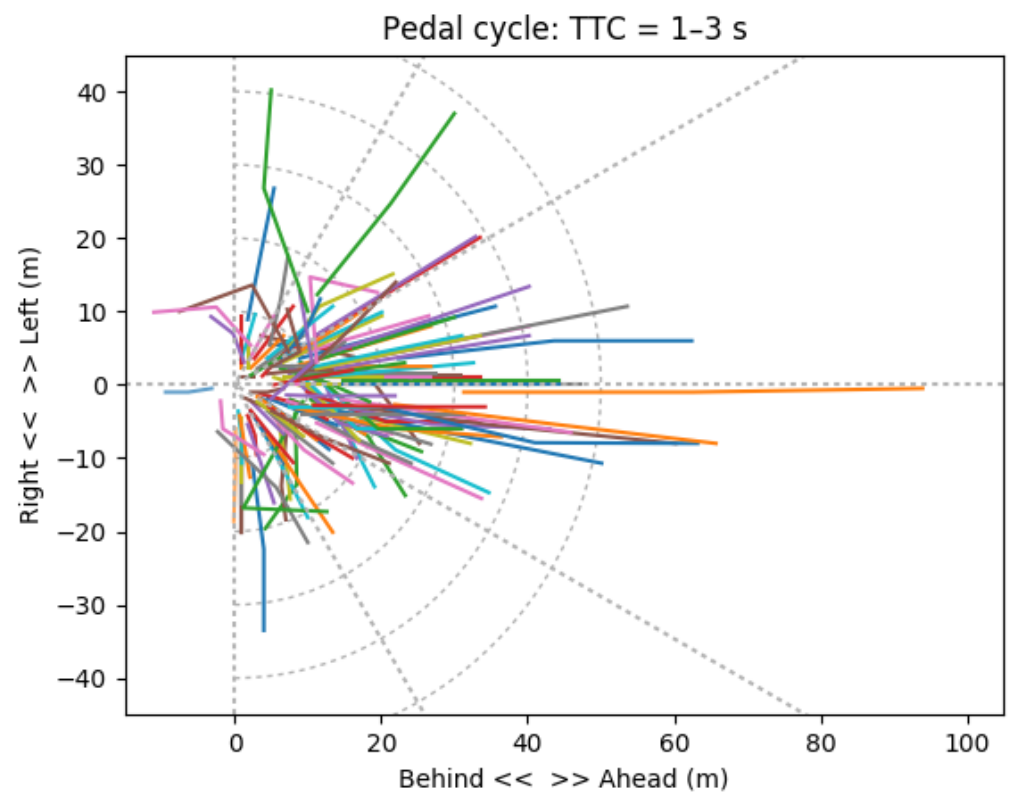

Figure 8: Position of bicycle relative to car at $\mathrm{TTC}=3,2,1 \mathrm{~s}$. 


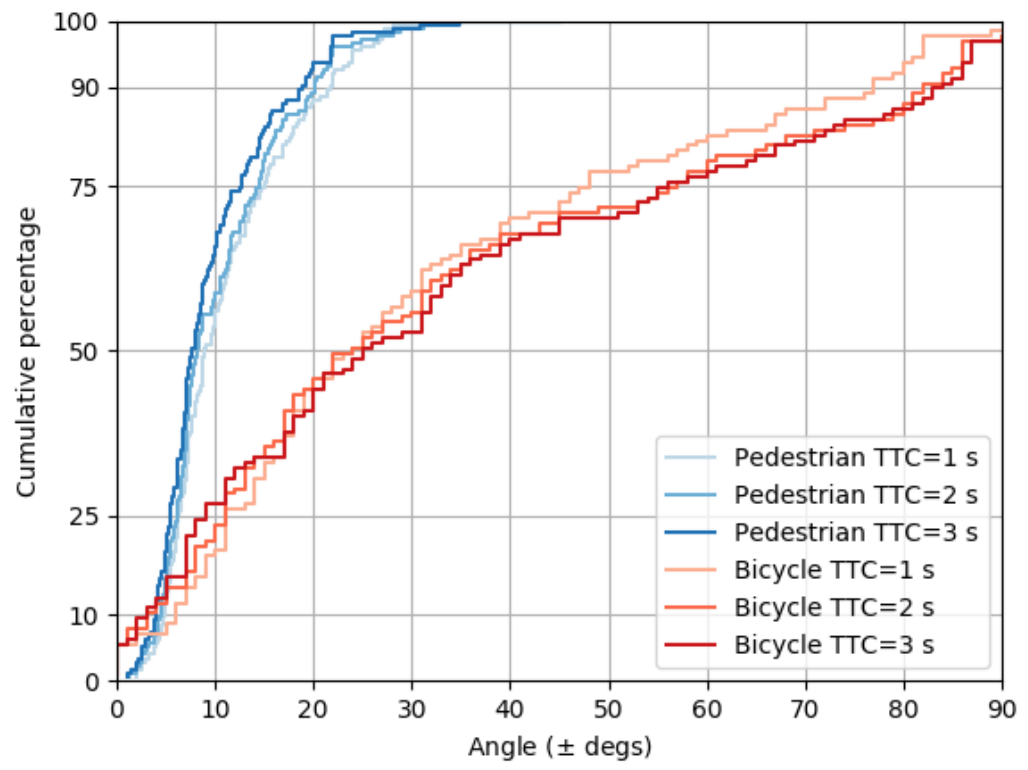

Figure 9: Angle of pedestrians and pedal cycles relative to car.

obscured cases were within around 55 degrees, while the 90th percentile was around 70 degrees for obscured cases and 80 degrees for visible cases. According to this crossover of the curves, obstructed cyclists were relatively more concentrated than visible cyclists at mid-range angles, around 30-60 degrees.

Figure 11 presents the distance of pedestrians and pedal cyclists away from the striking vehicle in the seconds before impact in the same manner that Figure 9 presents angle. For each group, as could be anticipated, longer time-to-collision is associated with greater distance: for example the median (50th percentile) distances for pedestrians were 11, 23 and 35 metres at 1,2 and 3 seconds respectively while for pedal cycles the distances were 8,15 and 22 metres. Similarly at the 75th percentile the distances were 13,26 and 39 metres for pedestrians and 10,21 and 32 metres for cyclists, while at the 90th percentile it was 16,32 and 50 metres and 14, 27 and 42 metres for pedestrians and cyclists respectively. The aspect of these results that was not predictable a priori is that pedal cyclists were generally closer to the striking vehicle than pedestrians at each given time-to-collision, as the numbers just cited confirm, i.e. in Figure 11 each curve for pedal cycles lies entirely to the left of the corresponding curve for pedestrians at the same time-to-collision. 


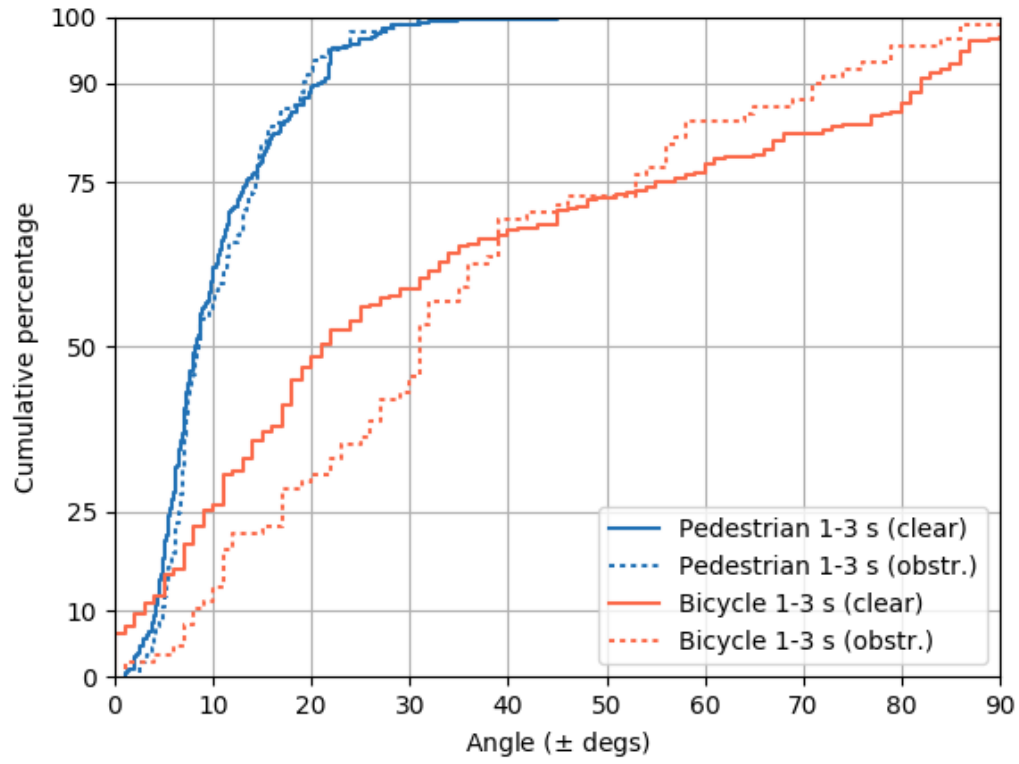

Figure 10: Angle of pedestrians and pedal cycles relative to car according to whether the driver's line of sight was clear or obstructed. 


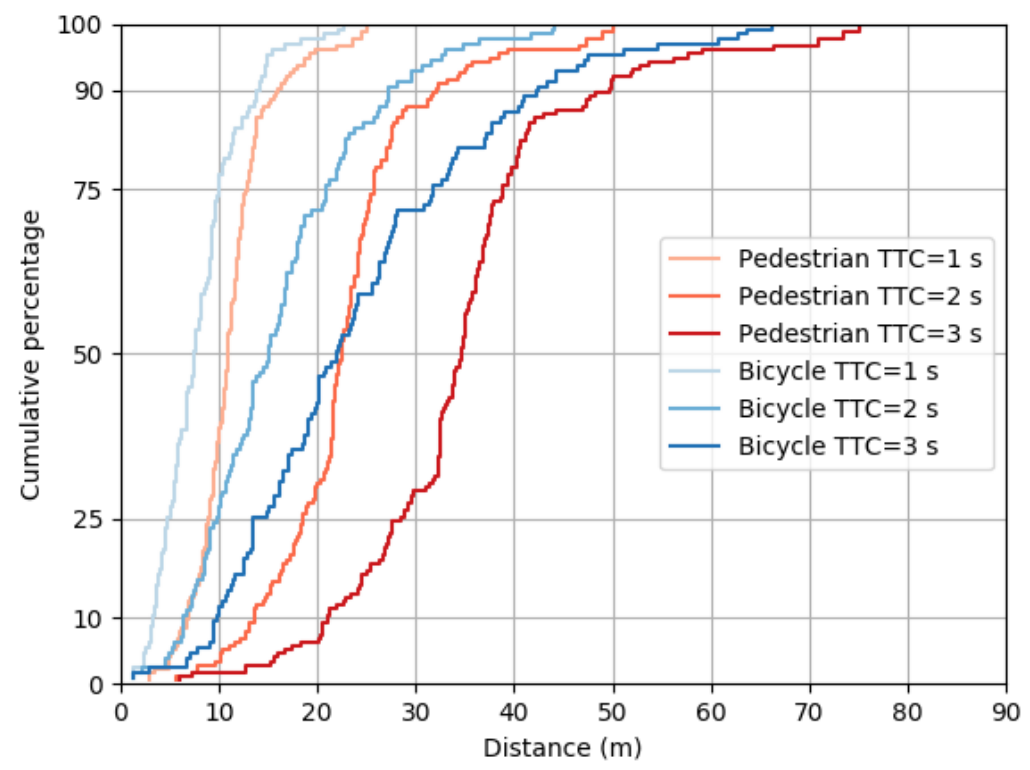

Figure 11: Distance of pedestrians and pedal cycles from car.

\section{Discussion}

The vehicle safety ratings provider EuroNCAP introduced an AEB test for pedestrians and can be expected to enhance this with a cyclist test in the future [17]. The pedestrian test features vehicle speeds of $20-60 \mathrm{~km} / \mathrm{h}$ and (lateral) pedestrian speeds of $5 \mathrm{~km} / \mathrm{h}$ and $8 \mathrm{~km} / \mathrm{h}$, including scenarios where the line of sight to the pedestrian is obstructed. The combination of the highest speeds, $60 \mathrm{~km} / \mathrm{h}$ and $8 \mathrm{~km} / \mathrm{h}$, produces the greatest distance between car and pedestrian in the seconds before impact, namely 17, 34 and 50 metres for 1, 2 and 3 seconds respectively. The most lateral angle is 22 degrees, produced by the lowest vehicle speed and the highest pedestrian speed, $20 \mathrm{~km} / \mathrm{h}$ and $8 \mathrm{~km} / \mathrm{h}$ respectively. Comparison with Figure 9 and Figure 10 reveals that these distances and angle are close to the 90th percentile for pedestrians. The pedestrian test is therefore quite demanding relative to the accident data considered in this study. To achieve the same level of coverage for pedal cyclists in a future test, the accident data-based on 90th percentile distances of 14, 27 and 42 metres at 1, 2 and 3 seconds before impact in Figure 11-suggests a closing speed of around $14 \mathrm{~m} / \mathrm{s}$ and a detection angle of 75-85 degrees from the longitudinal axis of the car.

The range and field of view for pedestrians observed in this study was consistent 


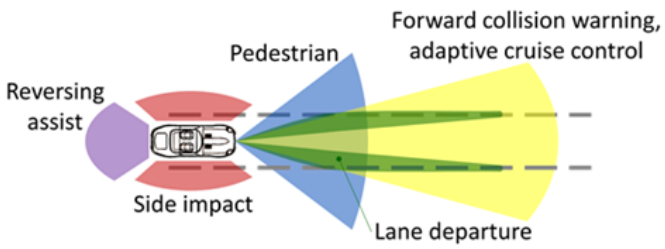

Figure 12: Examples of advanced safety and driver assistance systems in contemporary passenger cars.

with that reported on German in-depth data [6] judging, in the absence of percentile values, on a published graphical presentation of pedestrian location points. Less information is available for bicycle accidents. A review of severe cyclist casualties in eight European countries found England at the top end of male involvement in the range $55-83 \%$ and identified intersections as the site in $31-61 \%$ of cases [18]. The indepth sample in this study was consistent with the male involvement and featured $60 \%$ cross-intersections. It is possible that severe and fatal incidents may be more highly associated with head-on and rear-end impacts.

For the proportion of cases where the pedal cycle was located at an angle of around 40-50 degrees or more from the longitudinal axis of the car, it can be inferred that the speed of the car was comparable to or less than the speed of the bicycle. Many of these accidents were at cross intersections where the car had slowed down which is also consistent with the overall lower distance between cars and pedal cyclists (compared to pedestrians) described in the Results section. This was a point of dissimilarity between the two classes of vulnerable road users: unlike cyclists, pedestrians do not generally enter the intersecting area of two roadways. The length of pedal cycles along with their delicate state of balance and higher speeds are further points of dissimilarity that help explain why relatively slow moving cars played a role in a higher proportion of accidents.

The wide lateral scatter of pedal cycles raises the question of how technologically challenging it is to detect them. The advanced safety and driver assistance functions fitted to contemporary vehicles, some of which are illustrated in Figure 12, already provide a degree of surround vision. Technological developments in this area are proceeding very rapidly, not least by the drive towards autonomous vehicles which scan a full 360 degrees using a variety of short- and long-range sensors, including ultrasound, camera, radar and laser. A cyclist detection system [19] widely promoted in 2013 as the first production exemplar of its type employed a camera for image recognition and radar for distance assessment, similar to the detection of pedestrians and other vehicles. It cannot be forecast confidently whether extending the lateral field 
of view will involve additional sensors, modified (wide-angle) sensors, or different types of sensors. It is however clear that thinking about systems in isolation will make increasingly less sense: manufacturers are certain to integrate the advanced sensing technologies fitted to their vehicles and exploit their combined performance capability to achieve multiple safety and assistance functions.

The intention of this research was to contribute to the development and optimisation of effective AEB tests and in-vehicle systems for the protection of vulnerable road users. The circumstances of accidents involving pedestrians and pedal cycles vary from land to land according to many factors, including the density of these road users, the infrastructure provided for them to interact with powered road vehicles, local driving and road use culture, and characteristics of the on-road vehicle fleet. The results in this paper reflect the UK experience and are most likely to be transferable to countries or regions similar to the UK in relevant respects.

\section{Conclusions}

According to the in-depth, at-scene accident data considered in this report, the sensors used in autonomous emergency braking systems need to cover a much greater range of lateral angles for pedal cycles than for pedestrians, almost a full 180 degrees at 2-3 seconds before impact. On the other hand, despite the generally higher travel speed of cyclists compared to pedestrians, the detection range (distance) of the sensors that is appropriate for pedestrians should also suffice for pedal cycles.

\section{Acknowledgements}

The RAIDS-OTS project was funded by the Department for Transport and Highways Agency. Funding for analysis was received from Thatcham Motor Insurance Repair Research Centre (UK) and the Insurance Institute for Highway Safety (USA). The views expressed in this paper are those of the authors and are not intended to represent the views of any other individual or institution. The Department for Transport does not guarantee the accuracy or completeness of information inferred from its data and cannot accept liability for any loss or damages of any kind resulting from reliance on the information or guidance contained in this document.

\section{References}

[1] WHO, Global status report of road safety, on-line, accessed December 2017, World Health Organizaton (2015). 
URL http://ec.europa.eu/transport/road_safety/pdf/projects/ veronica_final_report.pdf

[2] M. Lindman, S. Jonsson, L. Jakobsson, T. Karlsson, D. Gustafson, A. Fredriksson, Cyclists interacting with passenger cars; a study of real world crashes, in: Proceedings of the International IRCOBI Conference on the Biomechanics of Impact, International Research Council on the Biomechanics of Injury, Lyon, 2015, pp. 1-12, IRC-15-10.

[3] G. Prati, M. D. Angelis, V. Marn-Puchades, F. Fraboni, L. Pietrantoni, Characteristics of cyclist crashes in Italy using latent class analysis and association rule mining, PLOS One 12 (2) (2017) 1-28.

[4] A. MacAlister, D. S. Zuby, Cyclist crash scenarios and factors relevant to the design of cyclist detection systems, in: Proceedings of the International Conference on the Biomechanics of Impact, International Research Council on the Biomechanics of Injury, Lyon, 2015, pp. 373-384, IRC-15-50.

[5] F. Leimbach, U. Schmortte, H. Kiebach, Global harmonisation of test procedures for driver assistance systems, in: Proceedings of the 23nd International Technical Conference on the Enhanced Safety of Vehicles, technical paper 13-0234, NHTSA, Seoul, 2013, pp. 1-8.

[6] E. Rosén, J.-E. Källhammer, D. Eriksson, M. Nentwich, R. Fredriksson, K. Smith, Pedestrian injury mitigation by autonomous braking, in: Proceedings of the 21 st International Technical Conference on the Enhanced Safety of Vehicles, technical paper 09-0132, NHTSA, Stuttgart, 2009, pp. 1-9.

[7] Y. Matsui, S. Oikawa, M. Hitosugi, Analysis of car-to-bicycle approach patterns for developing active safety devices, Traffic Injury Prevention 17 (4) (2016) 434439.

[8] R. J. Jansen, T. Lotan, M. Winkelbauer, J. Bärgman, J. Kovaceva, M. Donabauer, A. Pommer, O. Musicant, A. Harel, S. Wesseling, M. Christoph, N. van Nes, Interactions with vulnerable road users, EU FP7 project UDRIVE deliverable D44.1, European Commission, Brussels, FP7-SST-2012.4.1-3, GA no. 314050 (2018 forthcoming).

[9] C. A. Hobbs, P. F. Gloyns, S. J. Rattenbury, European new car assessment programme (EuroNCAP) — assessment protocol and biomechanical limits, Project report, Transport Research Laboratory, Wokingham, UK (May 1995). 
[10] R. Cuerden, M. Pittman, E. Dodson, J. Hill, The UK On-the-Spot accident data collection study_phase II report, Road Safety Research Report 73, Department for Transport, London (2008).

[11] DfT, Research database: Road Accident In-Depth Studies (RAIDS), on-line, accessed January 2018, Department for Transport (2013).

URL http: //www . dft.gov . uk/rmd/project . asp?intProjectID=12962

[12] DfT, Research database: On the Spot Accident Investigation Project phase 3, online, accessed January 2018, Department for Transport (2011).

URL http: //www . dft.gov.uk/rmd/project. asp?intProjectID $=12722$

[13] A. Schubert, C. Erbsmehl, L. Hannawald, Standardized pre-crash scenarios in digital format on the basis of the VFO simulation, on-line, accessed January 2018, Verkehrsunfallforschung an der TU Dresden GmbH (2009).

URL http://bast.opus.hbz-nrw.de/volltexte/2013/683/pdf/31_ Schubert_Erbsmehl.pdf

[14] R. Cuerden, M. McCarthy, The methodology and initial findings for the Road Accident In Depth Studies programme: RAIDS phase 1 report, Project report PPR808, Transport Research Laboratory (2016).

[15] J. Lenard, R. Danton, Accident data study in support of development of autonomous emergency braking test procedures, Report for Thatcham Research Centre and Insurance Institute of Highway Safety LUEL 5989/6175, Loughborough University (December 2010).

URL https://dspace.lboro.ac.uk/dspace-jspui/handle/2134/11769

[16] J. Lenard, A. Badea-Romero, R. Danton, Typical pedestrian accident scenarios for the development of autonomous emergency braking test protocols, Accident Analysis and Prevention 73 (2014) 73-80.

URL https://dspace.lboro.ac.uk/2134/21860

[17] EuroNCAP, AEB pedestrian, on-line, accessed January 2018, European New Car Assessment Program (2017).

URL https://www.euroncap.com/en/vehicle-safety/the-ratingsexplained/pedestrian-protection/aeb-pedestrian/

[18] L. T. Aarts, J. J. F. Commandeur, R. Welsh, S. Niesen, M. Lerner, P. Thomas, N. Bos, R. J. Davidse, Study on serious road traffic injuries in the EU, 
Tech. rep., European Commission, Brussels, contract no: MOVE/C4/SER/2015162/S12.714669 (2016).

[19] Volvo, Volvo Car Group reveals world-first cyclist detection with full auto brake in Geneva, on-line, accessed January 2018, Volvo Car Corporation (2013).

URL http://www.dft.gov.uk/rmd/project. asp?intProjectID $=12722$ 
Appendix 


\begin{tabular}{|c|c|c|c|c|c|c|c|c|}
\hline & $0-9$ & $10-19$ & $20-29$ & $30-39$ & $40-49$ & $50-100$ & Behind & Total \\
\hline $90-180$ & & & & & & & & 0 \\
\hline $60-89$ & & & & & & & & 0 \\
\hline $30-59$ & 1 & 1 & & & & & & 2 \\
\hline $0-29$ & 1 & 4 & 24 & 52 & 16 & 10 & & 107 \\
\hline $1-29$ & & 2 & 16 & 34 & 8 & 5 & & 65 \\
\hline $30-59$ & & 1 & & & & & & 1 \\
\hline $60-89$ & & & & & & & & 0 \\
\hline $90-179$ & & & & & & & & 0 \\
\hline Total & 2 & 8 & 40 & 86 & 24 & 15 & 0 & 175 \\
\hline \multicolumn{9}{|c|}{ With obstruction of view } \\
\hline $90-180$ & & & & & & & & 0 \\
\hline $60-89$ & & & & & & & & 0 \\
\hline $30-59$ & 0 & 1 & & & & & & 1 \\
\hline $0-29$ & 0 & 1 & 16 & 28 & 10 & 6 & & 61 \\
\hline $1-29$ & & 0 & 5 & 18 & 2 & 2 & & 27 \\
\hline $30-59$ & & 1 & & & & & & 1 \\
\hline $60-89$ & & & & & & & & 0 \\
\hline 90-179 & & & & & & & & 0 \\
\hline Total & 0 & 3 & 21 & 46 & 12 & 8 & 0 & 90 \\
\hline
\end{tabular}

\begin{tabular}{|c|c|c|c|c|c|c|c|c|}
\hline & $0-9$ & $10-19$ & $20-29$ & $30-39$ & $40-49$ & $50-100$ & Behind & Total \\
\hline $90-180$ & & & & & & & & 0 \\
\hline $60-89$ & & & & & & & & 0 \\
\hline $30-59$ & & 1 & & & & & & 1 \\
\hline $0-29$ & 5 & 27 & 61 & 10 & 5 & & & 108 \\
\hline $1-29$ & & 17 & 39 & 5 & & 3 & & 64 \\
\hline $30-59$ & & 2 & & & & & & 2 \\
\hline $60-89$ & & & & & & & & 0 \\
\hline 90-179 & & & & & & & & 0 \\
\hline Total & 5 & 47 & 100 & 15 & 5 & 3 & 0 & 175 \\
\hline \multicolumn{9}{|c|}{ With obstruction of view } \\
\hline $90-180$ & & & & & & & & 0 \\
\hline $60-89$ & & & & & & & & 0 \\
\hline $30-59$ & & 0 & & & & & & 0 \\
\hline $0-29$ & 4 & 13 & 25 & 1 & 2 & & & 45 \\
\hline $1-29$ & & 5 & 13 & 2 & & 0 & & 20 \\
\hline $30-59$ & & 1 & & & & & & 1 \\
\hline $60-89$ & & & & & & & & 0 \\
\hline $90-179$ & & & & & & & & 0 \\
\hline Total & 4 & 19 & 38 & 3 & 2 & 0 & 0 & 66 \\
\hline
\end{tabular}




\begin{tabular}{|c|c|c|c|c|c|c|c|c|}
\hline & $0-9$ & 10-19 & $20-29$ & $30-39$ & $40-49$ & $50-100$ & Behind & Total \\
\hline $90-180$ & & & & & & & & 0 \\
\hline 60-89 & & & & & & & & 0 \\
\hline $30-59$ & 1 & & & & & & & 1 \\
\hline $0-29$ & 40 & 63 & 5 & & & & & 108 \\
\hline $1-29$ & 24 & 38 & 3 & & & & & 65 \\
\hline $30-59$ & 1 & & & & & & & 1 \\
\hline $60-89$ & & & & & & & & 0 \\
\hline 90-179 & & & & & & & & 0 \\
\hline Total & 66 & 101 & 8 & 0 & 0 & 0 & 0 & 175 \\
\hline \multicolumn{9}{|c|}{ With obstruction of view } \\
\hline 90-180 & & & & & & & & 0 \\
\hline 60-89 & & & & & & & & 0 \\
\hline $30-59$ & 0 & & & & & & & 0 \\
\hline $0-29$ & 5 & 10 & 0 & & & & & 15 \\
\hline $1-29$ & 5 & 5 & 0 & & & & & 10 \\
\hline $30-59$ & 0 & & & & & & & 0 \\
\hline $60-89$ & & & & & & & & 0 \\
\hline $90-179$ & & & & & & & & 0 \\
\hline Total & 10 & 15 & 0 & 0 & 0 & 0 & 0 & 25 \\
\hline
\end{tabular}

\begin{tabular}{|c|c|c|c|c|c|c|c|c|}
\hline & $0-9$ & $10-19$ & $20-29$ & $30-39$ & $40-49$ & $50-100$ & Behind & Total \\
\hline 90-180 & & & & & & & 3 & 3 \\
\hline $60-89$ & 3 & 2 & 1 & & 1 & & & 7 \\
\hline $30-59$ & 2 & 7 & 4 & 2 & 1 & & & 16 \\
\hline $0-29$ & 2 & 5 & 10 & 8 & 7 & 3 & & 35 \\
\hline $1-29$ & 1 & 5 & 11 & 8 & 2 & 4 & & 31 \\
\hline $30-59$ & 2 & 8 & 5 & & & & & 15 \\
\hline 60-89 & & 10 & 7 & 1 & & & & 18 \\
\hline $90-179$ & & & & & & & 2 & 2 \\
\hline Total & 10 & 37 & 38 & 19 & 11 & 7 & 5 & 127 \\
\hline \multicolumn{9}{|c|}{ With obstruction of view } \\
\hline 90-180 & & & & & & & 1 & 1 \\
\hline 60-89 & 0 & 1 & 1 & & 0 & & & 2 \\
\hline $30-59$ & 1 & 4 & 2 & 2 & 0 & & & 9 \\
\hline $0-29$ & 0 & 1 & 2 & 3 & 1 & 0 & & 7 \\
\hline $1-29$ & 0 & 1 & 4 & 3 & 1 & 1 & & 10 \\
\hline $30-59$ & 0 & 2 & 3 & & & & & 5 \\
\hline $60-89$ & & 4 & 0 & 0 & & & & 4 \\
\hline 90-179 & & & & & & & 0 & 0 \\
\hline Total & 1 & 13 & 12 & 8 & 2 & 1 & 1 & 38 \\
\hline
\end{tabular}


Table 9: Position of bicycle relative to car at TTC $=2 \mathrm{~s}$ (cf. Figure 6).

\begin{tabular}{|c|c|c|c|c|c|c|c|c|}
\hline & $0-9$ & $10-19$ & $20-29$ & $30-39$ & $40-49$ & $50-100$ & Behind & Total \\
\hline $90-180$ & & & & & & & 2 & 2 \\
\hline $60-89$ & 4 & 3 & 1 & & & & & 8 \\
\hline $30-59$ & 7 & 4 & 2 & 1 & & & & 14 \\
\hline 0-29 & 3 & 19 & 14 & 2 & 1 & & & 39 \\
\hline $1-29$ & 3 & 13 & 9 & 3 & 2 & 1 & & 31 \\
\hline $30-59$ & 7 & 8 & & & & & & 15 \\
\hline $60-89$ & 5 & 10 & 1 & & & & & 16 \\
\hline $90-179$ & & & & & & & 2 & 2 \\
\hline Total & 29 & 57 & 27 & 6 & 3 & 1 & 4 & 127 \\
\hline \multicolumn{9}{|c|}{ With obstruction of view } \\
\hline $90-180$ & & & & & & & 1 & 1 \\
\hline $60-89$ & 0 & 1 & 0 & & & & & 1 \\
\hline $30-59$ & 3 & 2 & 2 & 0 & & & & 7 \\
\hline $0-29$ & 0 & 5 & 3 & 0 & 0 & & & 8 \\
\hline $1-29$ & 0 & 3 & 3 & 0 & 0 & 0 & & 6 \\
\hline $30-59$ & 1 & 3 & & & & & & 4 \\
\hline $60-89$ & 1 & 3 & 0 & & & & & 4 \\
\hline 90-179 & & & & & & & 0 & 0 \\
\hline Total & 5 & 17 & 8 & 0 & 0 & 0 & 1 & 31 \\
\hline
\end{tabular}

\begin{tabular}{|c|c|c|c|c|c|c|c|c|}
\hline & $0-9$ & $10-19$ & $20-29$ & $30-39$ & $40-49$ & $50-100$ & Behind & Total \\
\hline $90-180$ & & & & & & & & 0 \\
\hline 60-89 & 7 & & & & & & & 7 \\
\hline $30-59$ & 13 & 4 & & & & & & 17 \\
\hline 0-29 & 29 & 14 & 1 & & & & & 44 \\
\hline $1-29$ & 17 & 10 & 2 & 1 & & & & 30 \\
\hline $30-59$ & 13 & & & & & & & 13 \\
\hline $60-89$ & 12 & 1 & & & & & & 13 \\
\hline 90-179 & & & & & & & 3 & 3 \\
\hline Total & 91 & 29 & 3 & 1 & 0 & 0 & 3 & 127 \\
\hline \multicolumn{9}{|c|}{ With obstruction of view } \\
\hline $90-180$ & & & & & & & & 0 \\
\hline $60-89$ & 1 & & & & & & & 1 \\
\hline $30-59$ & 4 & 2 & & & & & & 6 \\
\hline $0-29$ & 4 & 1 & 0 & & & & & 5 \\
\hline $1-29$ & 2 & 1 & 0 & 0 & & & & 3 \\
\hline $30-59$ & 3 & & & & & & & 3 \\
\hline $60-89$ & 1 & 0 & & & & & & 1 \\
\hline 90-179 & & & & & & & 0 & 0 \\
\hline Total & 15 & 4 & 0 & 0 & 0 & 0 & 0 & 19 \\
\hline
\end{tabular}

